\title{
Assessment of two methods for the accurate prediction of transverse stress distributions in laminates
}

\author{
Johannes Främby ${ }^{\mathrm{a}, *}$, Jim Brouzoulis ${ }^{\mathrm{a}}$, Martin Fagerström ${ }^{\mathrm{a}}$ \\ ${ }^{a}$ Department of Applied Mechanics \\ Division on Material and Computational Mechanics \\ Chalmers University of Technology, Gothenburg, Sweden
}

\begin{abstract}
A major challenge for crash failure analysis of laminates is to find a modelling approach which is both sufficiently accurate and computationally efficient. We suggest to adopt a traditional single-layer shell formulation due to its cost effectiveness. In this contribution, we have therefore investigated the potential of two different concepts for obtaining better prediction of the through-the-thickness distribution of the transverse stresses; a crucial issue since the accuracy for a single-layer approach in this respect is normally low. The first concept is a multiscale approach in which the macroscopic shell model is concurrently coupled to a mesoscopic 3D element representation of the heterogeneous material structure on the laminate level. The second concept is a stress recovery method based on integration of the 3D equilibrium equations, with additional smoothing of the in-plane stresses.

The main conclusion drawn from the investigations is that, the adopted multiscale concept, although similar to what has been previously reported in the literature, is not a suitable approach to increase the level of accuracy of the predicted transverse stress distributions. However, we conclude that the proposed stress recovery method very well captures the through-the-thickness stress variations in our presented examples.
\end{abstract}

Keywords: stress recovery in shells, transverse stress prediction, shell to solid bridging

${ }^{*}$ Corresponding author. Address: Hörsalsvägen 7, SE-412 96 Göteborg, Sweden. Email: johannes.framby@chalmers.se. Phone: +46 317721300. 


\section{Introduction}

Numerical finite element (FE) tools for the accurate prediction of the crash response of vehicle structures in fibre reinforced plastics (FRP) are crucial for structural composites to have a widespread use in future cars [1]. Traditionally, FE crash simulations are performed using shell element models, which are well suited to model the thin-walled metal structures in automotive bodies while at the same time being computationally cost effective compared to continuum solid (3D) element models. However, a known drawback of traditional shell element formulations is low accuracy of the through-thethickness variation of the transverse stress components [2]. Thus, to be able to have a good level of predictability when simulating progressive crash failure in FRPs (e.g. to capture delamination, driven by high transverse stresses), better suited types of FE-models, than those traditionally used to model metals, need to be adopted.

A major challenge for crash failure analysis of laminates is thereby to find a modelling approach which is both sufficiently accurate and computationally efficient - a challenge addressed in this paper. Seeking a good compromise, we note that methods for simulating the structural behaviour of a laminated structure in a FE-framework can, following Reddy [2], generally be divided into two categories. Either layer-wise models (LWM), where each ply (or ply interface) of the laminate is represented by separate degrees of freedom $(\mathrm{DoF})^{1}$, or equivalent single-layer models (ESLM) where one layer of shell elements is used to represent the entire laminate. In the review by Carrera [3], it was concluded that the accuracy of the transverse stresses in LWM were superior compared to ESLM and that mixed formulations, where e.g. the transverse stress components can be regarded as unknown DoF, showed superior accuracy compared to traditional pure displacement type ones. Please refer also the review on Reissner Mixed Variational Theorem (RMVT) by Carrera [4], where a unified formulation is introduced, and the general review on modelling of FRP laminates by Kreja [5]. On the other hand, ESLM are more computationally efficient compared to LWM and if the accuracy of the transverse stresses can be improved, they will be highly competitive.

The subject of improving the transverse stresses in ESLM have been addressed by many authors over the years. Recently the work by Carrera and

\footnotetext{
${ }^{1}$ This category includes the $3 \mathrm{D}$ element models and the stacked shell element models, where each ply is modelled with at least one layer of elements in the thickness direction.
} 
co-workers was extended to construct an advanced locking-free finite element based on the RMVT formulation [6]. Besides adopting such a priori assumptions like mixed formulations or Zig-Zag in the shell formulation (see e.g. the historical review by Carrera [7]), a posteriori methods can be adopted [3].

All in all, and despite the conclusions made by Carrera [3] we suggest to adopt an ESLM shell formulation due to its cost effectiveness compared to LWM. The main argument for this is that LWM would for many real laminates require a too large amount of DoF which would be directly inappropriate for crash or other large scale analyses.

Therefore, the main goal of this work has been to establish a robust modelling method, which benefits from the cost efficiency of an ESLM, but at the same time yields accurate predictions of the transverse stresses. As an initial step, we will in this paper focus on the transverse shear stresses.

For this purpose, two methods have been implemented and assessed in terms of accuracy of the transverse shear stress distribution. First, we have investigated the potential of using a multiscale approach as a possible remedy to the problem of using ESLM stated above. A long term idea of adopting such an approach is to enable a model-adaptivity procedure, $c f$. e.g. Oden and Vemaganti [8], where initially the model is build up as an ESLM. Based on some measure, either a model error estimator or a failure initiation criterion, a transition to a coupled multiscale approach could be made locally in critical areas. In particular, we have adopted the multiscale concept introduced by Larsson and Landervik [9] for simulating deformations of thin-walled porous structures by coupling the macroscopic shell model to a mesoscopic 3D element representation of the heterogeneous material structure. Due to their promising results, our intention in this paper has been to investigate if a similar procedure can be adopted for simulating progressive failure in a laminated FRP plate. The main conclusion drawn from the investigations presented in this paper is however that, the concept proposed in [9] is not a suitable approach to increase the level of accuracy of the predicted transverse stress distributions.

As an alternative method, we have identified a suitable, and seemingly robust, post-processing procedure which allows accurate predictions of the transverse stress distribution to be made. This procedure is based on a nodal recovery of the in-plane stress components, averaged over neighbouring elements, followed by an integration of the transverse stress components using the 3D equilibrium equations. These recovered stresses can then be used in an initiation criterion for interlaminar crack nucleation, after which the 
delamination can be modelled using e.g. an appropriate cohesive formulation.

\subsection{Outline of paper}

First we set out to describe the adopted shell formulation in Section 2 together with a motivation of this particular choice. In Section 3, we continue by presenting the kinematics and choice of boundary conditions for the mesoscopic model necessary as part of the multiscale approach. Then in Section 4, our post-processing procedure is described and in Section 5 numerical examples are presented, which compare results from both the multiscale and the post-processing method. Detailed studies illustrating the effect from choosing different boundary conditions and RVE sizes are presented for an isotropic and a laminated cantilever beam. Finally, conclusions and discussions thereon are presented in Section 6.

\section{Shell formulation}

In this section, we will describe the underlying shell element formulation adopted in the current work, which is identical to what was proposed by Larsson and Landervik [9]. Thus, we adopt a solid-like ESLM shell formulation based on first-order shear deformation theory (FSDT) with a second-order expansion of the deformed configuration in the normal direction leading to a 7-parameter displacement formulation. The main ingredients of this formulation is repeated below, where in the subsequent text we let Latin letters denote the range from 1 to 3 and Greek letters denote the range from 1 to 2 .

\subsection{Reference and current shell geometry in terms of convected coordinates}

As a staring point, the undeformed (reference) configuration $\mathcal{B}_{0}$ of the shell is considered parametrised in terms of convected coordinates $\boldsymbol{\xi}$ as

$$
\mathcal{B}_{0}=\left\{\boldsymbol{X}:=\boldsymbol{\Phi}(\boldsymbol{\xi})=\boldsymbol{\Phi}_{0}\left(\boldsymbol{\xi}_{0}\right)+\xi \boldsymbol{M}\left(\boldsymbol{\xi}_{0}\right): \boldsymbol{\xi}_{0} \in A, \xi \in \frac{h_{0}}{2}[-1,1]\right\}
$$

where we introduced the compact notations $\boldsymbol{\xi}=\left(\xi^{1}, \xi^{2}, \xi^{3}=\xi\right)$ and $\boldsymbol{\xi}_{0}=$ $\left(\xi^{1}, \xi^{2}\right)$ and where the mapping $\boldsymbol{\Phi}$ maps the inertial Cartesian frame into the undeformed configuration as shown in Figure 1. In Eq. (1), the mapping

$\boldsymbol{\Phi}$ is defined by the midsurface placement $\boldsymbol{\Phi}_{0}$ and the outward unit normal director field $\boldsymbol{M}$. The coordinate $\xi$ is associated with the normal director field, $h_{0}$ is the initial thickness of the shell and $A$ is the midsurface area. 
Associated with the mapping $\boldsymbol{\Phi}$, we also have the covariant basis vectors $\boldsymbol{G}_{i}$ in the reference configuration expressed as

$$
\boldsymbol{G}_{i}=\boldsymbol{\Phi}_{, i}
$$

where $\bullet_{, i}$ denotes the derivative of $\bullet$ with respect to $\xi^{i}$. From Eq. (1) $\boldsymbol{G}_{i}$ are given as

$$
\begin{aligned}
\boldsymbol{G}_{\alpha} & =\boldsymbol{\Phi}_{0, \alpha}+\xi \boldsymbol{M}_{, \alpha} \\
\boldsymbol{G}_{3} & =\boldsymbol{G}^{3}=\boldsymbol{M} .
\end{aligned}
$$

Similar to Eq. (1), the deformed (current) configuration $\mathcal{B}$ is described as

$$
\mathcal{B}=\left\{\boldsymbol{x}:=\boldsymbol{\varphi}(\boldsymbol{\xi})=\boldsymbol{\varphi}_{0}\left(\boldsymbol{\xi}_{0}\right)+\xi \boldsymbol{m}\left(\boldsymbol{\xi}_{0}\right)+\frac{1}{2}(\xi)^{2} \gamma\left(\boldsymbol{\xi}_{0}\right) \boldsymbol{m}\left(\boldsymbol{\xi}_{0}\right)\right\}
$$

where the mapping $\boldsymbol{\varphi}$ is defined by the midsurface placement $\boldsymbol{\varphi}_{0}$, the spatial director field $\boldsymbol{m}$ and an additional scalar thickness inhomogeneity strain $\gamma$, $c f$. also Figure 1. As can be seen, the current configuration corresponds to a second order Taylor expansion along the director field, involving the inhomogeneity strain $\gamma$, thereby describing inhomogeneous thickness deformation effects of the shell. In this fashion we can avoid the pathological Poisson locking effect. If we consider a relative motion $\mathrm{d} \boldsymbol{x}$ of the non-linear placement $\boldsymbol{\varphi}$ as

$$
\mathrm{d} \boldsymbol{x}=\left(\boldsymbol{\varphi}_{0, \alpha}+\boldsymbol{m}_{, \alpha}\left(\xi+\frac{1}{2} \gamma \xi^{2}\right)+\frac{1}{2} \gamma_{, \alpha} \xi^{2} \boldsymbol{m}\right) \mathrm{d} \xi^{\alpha}+\boldsymbol{m}(1+\gamma \xi) \mathrm{d} \xi,
$$

the covariant basis vectors $\boldsymbol{g}_{i}=\boldsymbol{\varphi}_{, i}$ in the deformed configuration become

$$
\begin{aligned}
\boldsymbol{g}_{\alpha} & =\boldsymbol{\varphi}_{0, \alpha}+\left(\xi+\frac{1}{2} \gamma \xi^{2}\right) \boldsymbol{m}_{, \alpha}+\frac{1}{2} \gamma_{, \alpha} \xi^{2} \boldsymbol{m} \\
\boldsymbol{g}_{3} & =(1+\gamma \xi) \boldsymbol{m} .
\end{aligned}
$$

The pertinent deformation gradient $\boldsymbol{F}$, to the adopted kinematics is obtained as

$$
\mathrm{d} \boldsymbol{x}=\boldsymbol{F} \cdot \mathrm{d} \boldsymbol{X} \text { with } \boldsymbol{F}=\frac{\partial \boldsymbol{x}}{\partial \xi^{i}} \otimes \frac{\partial \xi^{i}}{\partial \boldsymbol{X}}=\boldsymbol{g}_{i} \otimes \boldsymbol{G}^{i}
$$

where the contravariant basis vectors in the reference configuration $\boldsymbol{G}^{i}$ are obtained from the covariant basis vector by

$$
\boldsymbol{G}^{j}=G^{i j} \boldsymbol{G}_{i},
$$




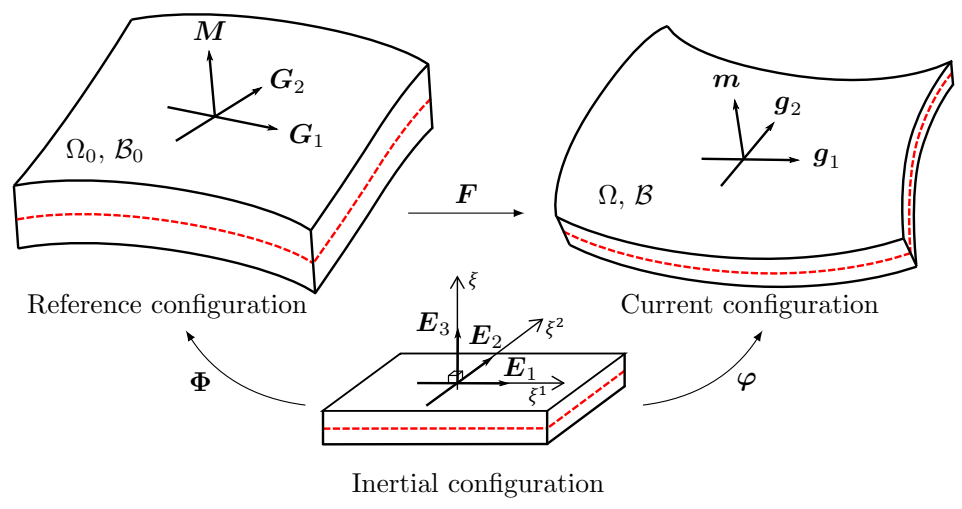

Figure 1: Mappings of shell model defining undeformed and deformed shell configurations relative to inertial Cartesian frame.

using the metric tensor defined as

$$
G_{i j}=\boldsymbol{G}_{i} \cdot \boldsymbol{G}_{j} \text { and } G^{i j}=\left(G_{i j}\right)^{-1} .
$$

This kinematic representation is then inserted in the (static) momentum balance to obtain the resulting shell element formulation, $c f$. [9] for details.

\section{Mesoscopic laminate model}

In the multiscale modelling concept introduced by Larsson and Landervik [9], the momentum balance is set up as an initial boundary value problem using a shell element formulation on the (component) macroscale. The heterogenic mesoscopic (laminate) level is then explicitly accounted for via nested FE analyses of a Representative Volume Element (RVE) modelled using 3D elements ( $c f$. e.g. Kouznetsova et al. [10]) providing the homogenised stress resultants in each integration point of the shell surface. We acknowledged that similar multiscale methods have been applied to FRP laminated plates also by other authors, e.g. by Coenen et al. [11] and by Helfen and Diebels [12], however the focus therein have been other than accuracy of the through-the-thickness stress variation.

Emphasis in the current contribution is put on assessing the potential of this method for obtaining accurate stress distributions in the laminate by means of multiscale (or two-scale) modelling. For this purpose, we focus only on the prolongation conditions, i.e. the link from the macroscale shell problem to the laminate level RVE analysis, which is detailed in the subsequent 
section. For further details about the associated homogenisation procedure interested readers are referred to [9].

In the following, we denote quantities related to the macroscopic fields by a superimposed bar, e.g.

\subsection{Kinematic expansions of macroscopic fields in the RVE}

In order to couple the macroscopic and the mesoscopic fields in the prolongation step to the RVE, Larsson and Landervik [9] suggest to use different Taylor series expansions for the deformation map with respect to the tangent plane and thickness direction of the shell. Hence, we express the finite mesoscopic placement $\Delta \boldsymbol{x}[\boldsymbol{X}]$ (measured as the placement relative to the expansion point $\overline{\boldsymbol{X}}$ ) within the RVE in separate portions in the tangent- and the thickness directions, denoted by $\Delta \overline{\boldsymbol{x}}_{=}$and $\Delta \overline{\boldsymbol{x}}_{\perp}$, respectively, $c f$. Figure 2 . Together with the mesoscopic displacement field $\boldsymbol{u}_{\mathrm{f}}$, we express the relative placement $\Delta \boldsymbol{x}[\boldsymbol{X}]$, following [9], as

$$
\Delta \boldsymbol{x}=\Delta \overline{\boldsymbol{x}}_{=}+\Delta \overline{\boldsymbol{x}}_{\perp}+\boldsymbol{u}_{\mathrm{f}}
$$

with a first order expansion in the tangent direction

$$
\Delta \overline{\boldsymbol{x}}_{=}=\overline{\boldsymbol{F}}_{\mid \xi} \cdot \Delta \boldsymbol{X}_{=}=\overline{\boldsymbol{g}}_{\alpha \mid \xi} \Delta \xi^{\alpha} \text { with } \Delta \boldsymbol{X}_{=}=\overline{\boldsymbol{G}}_{\alpha} \Delta \xi^{\alpha}
$$

where the notation $\bullet \xi$ implies that the expansion is made for a fixed value of $\xi$. In the thickness direction, a second-order expansion is made about the origin of the RVE

$$
\Delta \overline{\boldsymbol{x}}_{\perp}=\overline{\boldsymbol{F}}_{\mid \xi=0} \cdot \Delta \boldsymbol{X}_{\perp}+\frac{1}{2}\left(\overline{\boldsymbol{K}}_{\mid \xi=0} \cdot \Delta \boldsymbol{X}_{\perp}\right) \cdot \Delta \boldsymbol{X}_{\perp}=\overline{\boldsymbol{g}}_{3 \mid \xi=0} \xi+\frac{1}{2} \overline{\boldsymbol{g}}_{3,3 \mid \xi=0} \xi^{2}
$$

which involves the second gradient $\overline{\boldsymbol{K}}=\left(\overline{\boldsymbol{g}}_{i} \otimes \overline{\boldsymbol{G}}^{i}\right)_{, j} \otimes \overline{\boldsymbol{G}}^{j}$. Please note that with this choice of expansion through the thickness, the shell curvature is only partially accounted for. The consequence of this is discussed in relation to the numerical examples below.

In view of the relations Eq. (12)-Eq. (14), we obtain the kinematics of the RVE in terms of the generalised shell strain measures as

$$
\Delta \boldsymbol{x}=\overline{\boldsymbol{g}}_{\alpha \mid \xi} \Delta \xi^{\alpha}+\overline{\boldsymbol{m}}\left(\xi+\frac{1}{2} \bar{\gamma}(\xi)^{2}\right)+\boldsymbol{u}_{\mathrm{f}}[\boldsymbol{X}]
$$




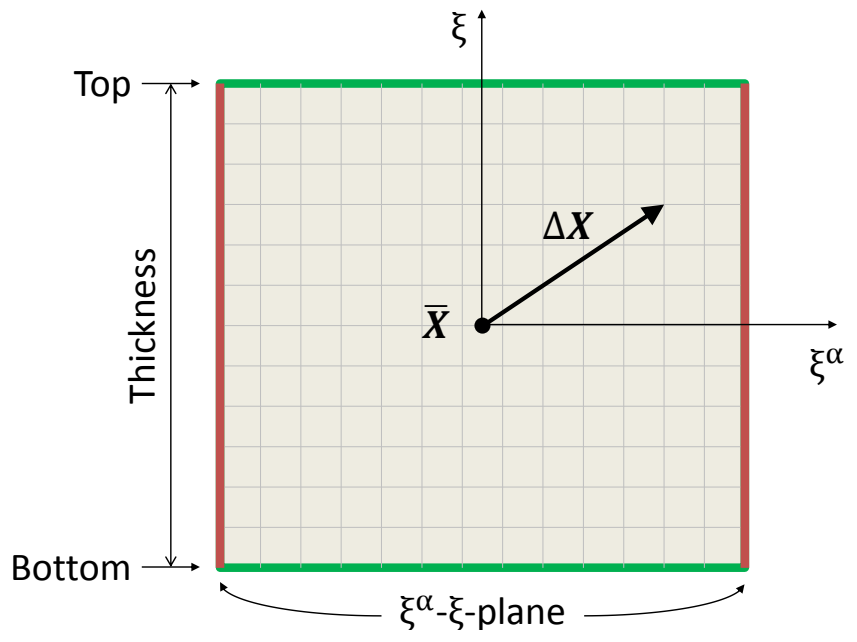

Figure 2: Sketch of midsection through the $12 \times 13 \times 13$ (hexahedra) element RVE showing the expansion point $\overline{\boldsymbol{X}}$ and the placement vector $\Delta \boldsymbol{X}$ in the reference configuration.

The mesoscopic deformation gradient $\mathrm{d} \Delta \boldsymbol{x}=\boldsymbol{F} \cdot \mathrm{d} \boldsymbol{X}$ associated with this kinematic description, while considering that $\xi$ is fixed in Eq. (13), now finally becomes

$$
\boldsymbol{F}=\left(\overline{\boldsymbol{g}}_{i}+\frac{\partial \boldsymbol{u}_{f}}{\partial \xi^{i}}\right) \otimes \overline{\boldsymbol{G}}^{i}=\overline{\boldsymbol{F}}+\boldsymbol{H}_{f} \text { with } \overline{\boldsymbol{F}}=\overline{\boldsymbol{g}}_{i} \otimes \overline{\boldsymbol{G}}^{i} \text { and } \boldsymbol{H}_{f}=\frac{\partial \boldsymbol{u}_{\mathrm{f}}}{\partial \boldsymbol{X}}
$$

In practical terms, the prolongation of the multiscale model is performed by extracting the generalised strains, i.e. $\overline{\boldsymbol{g}}_{\alpha \mid \xi}, \overline{\boldsymbol{m}}$ and $\bar{\gamma}$, from the macroscopic shell model such that the proper boundary displacements can be computed and applied to the RVE via Eq. (15). We will in the next section describe the RVE boundary conditions associated with this step.

As to the the size of the RVE, we note that its thickness is given by the thickness of the plate, whereas the in-plane breadth and width of the RVE should be chosen based on a balance between accuracy and computational efficiency as exemplified later in the numerical section.

\subsection{Application of boundary conditions}

In [9] the prolongation from macroscopic to mesoscopic level was performed using a combination of Dirichlet and Neumann boundary conditions. More specifically, the in-plane surfaces of the RVE were prescribed to the macroscopic generalised shell strain using a Dirichlet boundary conditions 
such that the fluctuation field $\mathbf{u}_{\mathrm{f}}$ is prescribed to vanish on these surfaces. On the remaining top and bottom surfaces, Neumann (traction free) boundary conditions were applied, $c f$. Figure 3a. This type of boundary conditions, which we in the sequel will denote as Mixed boundary conditions, will result in energy equivalence across the scales. However, since $\mathbf{u}_{\mathrm{f}}$ is unrestricted on the top and bottom these boundary conditions do not guarantee that the average deformation is maintained. Concerns regarding this potential problem will be raised in the following sections.

For comparison, we will also investigate boundary conditions that will ensure deformation equivalence across the scales, e.g. the classical Dirichlet boundary condition. This can be achieved using pure Dirichlet boundary conditions, denoted Dirichlet in the sequel, where all six surfaces of the RVE are subjected to the condition $\mathbf{u}_{\mathrm{f}}=\mathbf{0}, c f$. Figure $3 \mathrm{~b}$. Clearly this is a more constrained case and the assumption is that this choice of boundary conditions will yield a solution much closer to what is obtained from a pure shell analysis. As a final comparison, we will also investigate the response obtained for a Taylor assumption, i.e. the case where the fluctuation field $\mathbf{u}_{\mathrm{f}}$ is set to zero in the entire domain (denoted Taylor boundary conditions) as shown in Figure 3c.

\section{Stress recovery of transverse stresses}

Over the years different methods to make a posteriori evaluation of the transverse stresses in FE elements have been developed. These post-processing methods are typically based on Hooke's law, the 3D equilibrium equations, simplifying assumptions [13] or predictor-corrections approaches [14], see e.g. the reviews by Carrera [3] and Noor and Malik [15]. Although the recovery of the transverse stresses from the $3 \mathrm{D}$ equilibrium equations is a rather simple method $^{2}$, both reviews concluded that, for mechanical loading, it provides acceptable results. The fundamentals for this method, which can be found in e.g. Kant and Manjunatha [16] or Park et al. [17], are described below.

\footnotetext{
${ }^{2}$ To the best of our knowledge, it dates back to (at least) the early work by Dmitrii Ivanovich Zhuravskii who used it to derive the formula for shear stresses during bending in 1855 .
} 


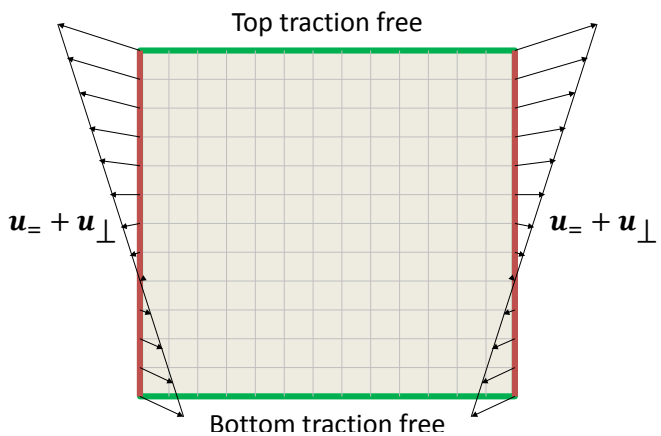

(a)

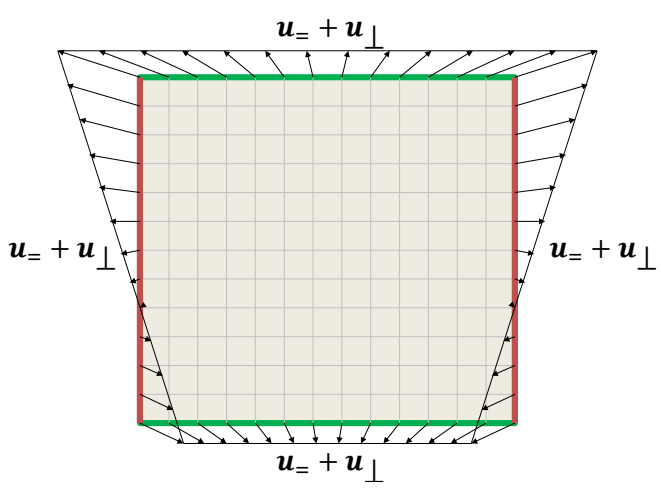

(b)

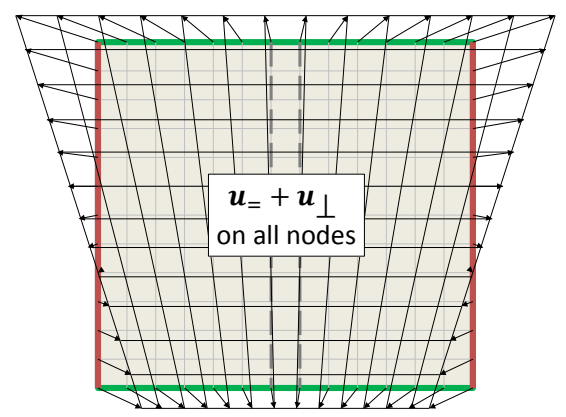

(c)

Figure 3: Prescribtion of the fluctuation $\mathbf{u}_{\mathrm{f}}$ on the edges of the RVE according to the Mixed (a), Dirichlet (b) and Taylor (c) boundary conditions. 


\subsection{The proposed stress recovery method}

The transverse stresses can be determined from the in-plane stresses by using the $3 \mathrm{D}$ equilibrium equations

$$
\sigma_{i j, j}+f_{i}=\rho \ddot{u}_{i}
$$

where $\sigma_{i j}$ are the Cauchy stress components, $f_{i}$ are the body forces, $\rho \ddot{u}_{i}$ are the inertia effects and $\bullet, j$ denotes the derivative with respect to local laminate coordinate $x_{j}$.

In the static case and for zero body forces, we can recover the transverse stress components at the top interface of ply $k$ ( $c f$. Figure 4) by integration from the bottom of the laminate as:

$$
\hat{\sigma}_{i 3}^{k}=-\sum_{n=1}^{k} \int_{x_{3}^{n-1}}^{x_{3}^{n}}\left(\sigma_{1 i, 1}+\sigma_{2 i, 2}\right) \mathrm{d} x_{3}+C_{i},
$$

where $x_{3}$ is the the transverse direction and $\hat{\bullet}$ denotes recovered values. In Eq. (18), the integration constants $C_{i}$ are determined from the known values of $\sigma_{i 3}$ at either of the two boundaries at $x_{3}= \pm \frac{h}{2}$, see e.g. Kant and Manjunatha [16]. Since the transverse normal stress $\hat{\sigma}_{33}$ is determined from the in-plane gradients of the transverse shear components $\sigma_{\alpha 3, \alpha}$, we perform the recovery of the transverse stresses in two steps. First the transverse shear stresses $\hat{\sigma}_{\alpha 3}$ are computed from the in-plane stress gradients $\sigma_{\alpha \beta, \alpha}$. Then in a second step the transverse normal stress $\hat{\sigma}_{33}$ is computed from the recovered transverse shear stresses $\hat{\sigma}_{\alpha 3, \alpha}$.

To perform the integration in Eq. (18), the gradient of the in-plane stresses must first be extracted from the FE-solution. We choose to do this by approximating the stress variation using a quadratic interpolation over a wedge, cf. Figure 4. By performing a linear least square fit (LSF), based on the known stress values in Gauss points of the current ply, we can determine the nodal values of the wedge interpolant. When the stress values at each wedge node has been determined, the ply-by-ply integration across the thickness in Eq. (18) can be performed by numerical integration. We do this at all in-plane positions of the Gauss points of the element.

Please note that the stress recovery method presented above involves the in-plane stress gradients, which must be properly evaluated in order to obtain accurate results. With the chosen shell kinematics, the in-plane stress 

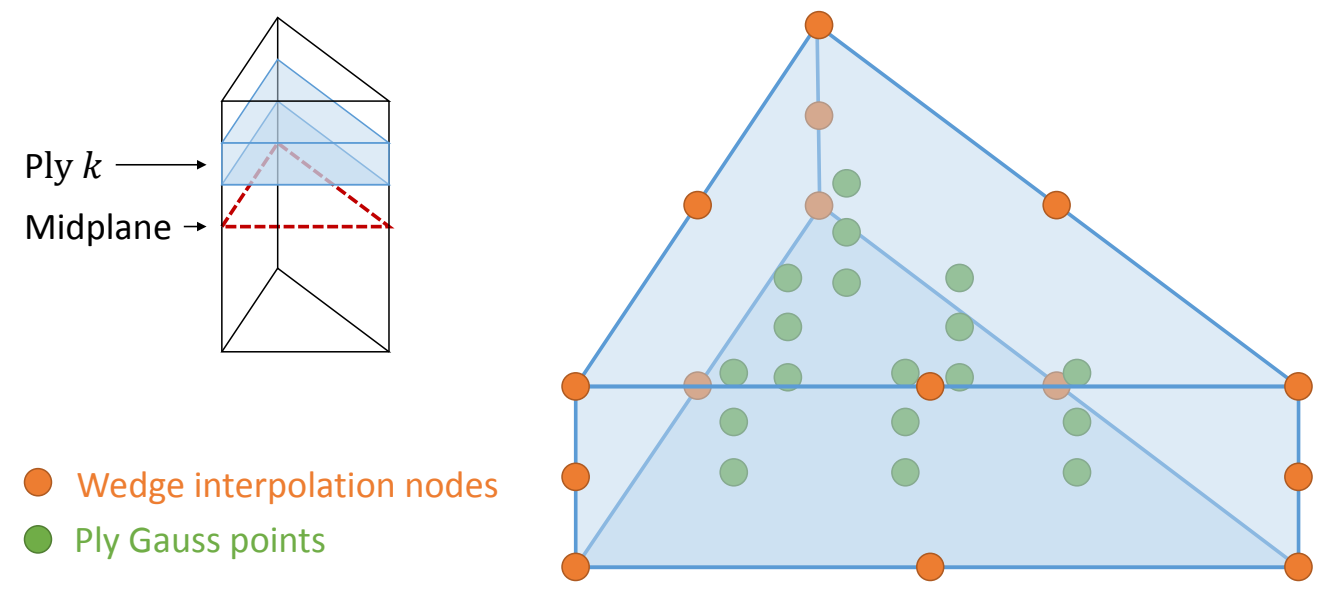

Figure 4: Sketch of wedge interpolation element used to extract stress values from the ply Gauss points to the ply wedge nodes using a least square fit.

components themselves are predicted with acceptable accuracy, as demonstrated in the numerical examples in Section 5. However, as in the case of any $C_{0}$-continuous finite element, these stresses from the $\mathrm{FE}$ solution are not continuous over element boundaries. Thus, it is not sufficient to construct the stress interpolation individually within each element.

In order to improve the results, we introduce an intermediate smoothing step to create a continuous stress field over the element boundaries. This smoothing step can be done in several ways, e.g. simple stress averaging across elements, adopting the well known super convergent patch recovery method by Zienkiewicz and Zhu [18] or more recent methods given by Payen and Bathe [19] and by Bush et al. [20]. In this work we use a method where the stress values at each wedge node are average values obtained from the adjacent wedge elements in each ply, cf. Figure 5.

\section{Numerical examples}

To asses the performance of the multiscale approach and the stress recovery procedure, in terms of accuracy in the resulting stress distributions, we present two numerical examples below. First in Subsection 5.2, we study an isotropic laminate since this allows us to make comparisons with analytical solutions. Then, in Subsection 5.3, we make an assessment using an angle-ply 

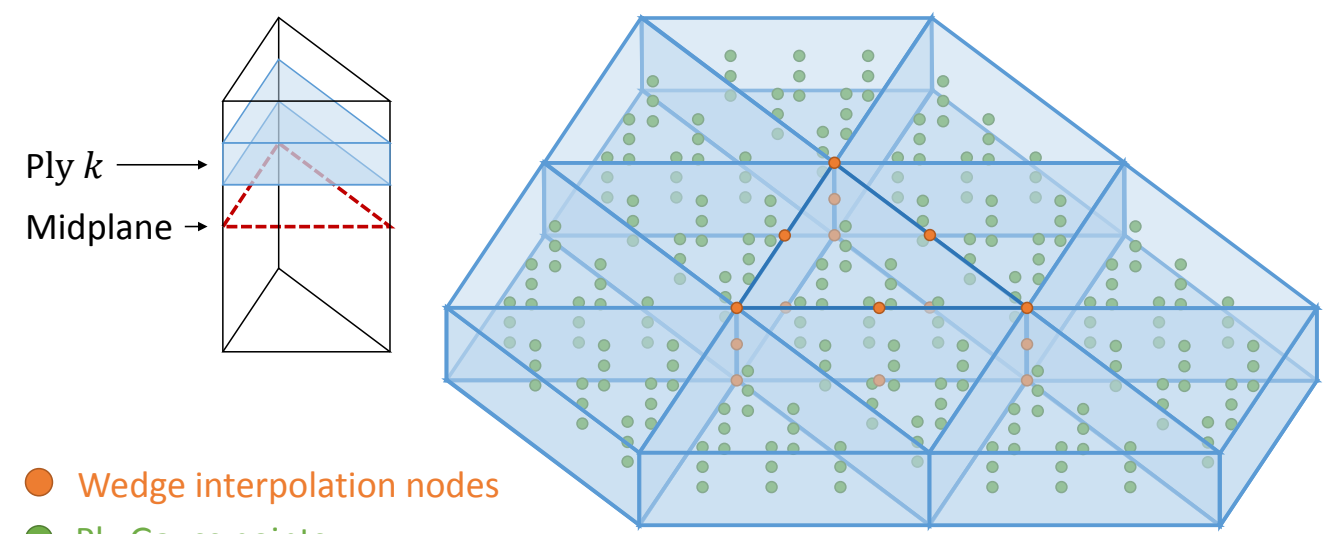

Wedge interpolation nodes

Ply Gauss points

Figure 5: Sketch of stress extraction where the value of the wedge nodes are average values of LSF from all neighbouring wedge interpolation elements connected to the specific node.

laminated plate. For the multiscale approach, we focus on investigating the effects of varying the in-plane size of the mesoscopic RVE and the effects from applying different types of boundary conditions in the prolongation phase, as described in Section 3.2.

In all our numerical examples we consider a square plate, clamped along one edge and subjected to a prescribed displacement of $\delta=0.1 \mathrm{~mm}$ on the opposite edge, $c f$. Figure 6.

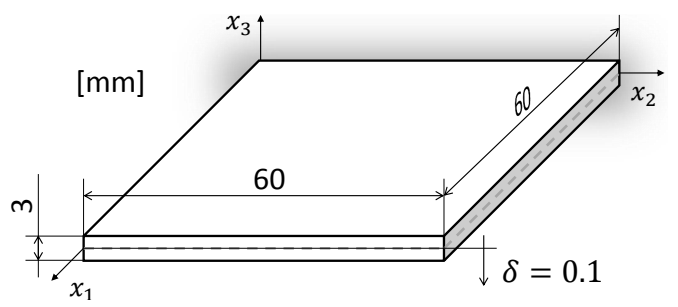

Figure 6: Sketch of the numerical example. $N B$ : proportions not representative.

\subsection{Preliminaries}

As mentioned previously, we focus on the prolongation phase of the multiscale approach. Thus, the deformation of the plate (extracted from the 
macroscopic shell simulation) is applied to the RVE as described in Section 3 and we then solve for the stresses in the RVE. Furthermore, to avoid macroscopic boundary effects, we choose to evaluate the stresses at a point close to the middle of the plate.

The (macroscopic) shell model consists of 450 isosceles right second order triangle elements ( $4 \mathrm{~mm}$ mesh size, $c f$. Figure 7a), while the RVE is modelled with cubic shaped linear hexahedron elements $(0.25 \mathrm{~mm}$ mesh size giving 12 elements through the thickness).

The in-plane dimensions of the studied RVEs are varied in three steps from $1.25 \mathrm{~mm}$ (5 elements) to $6.25 \mathrm{~mm}$ (25 elements), cf. Figure 8. In each RVE, the stress profiles are extracted by taking the in-plane average of all element integration points at each given $\xi$-level (thickness coordinate).

The obtained stress distributions are then compared to those obtained from the stress recovery method and the pure shell model (using the proposed 7-parameter shell element), with a 3D continuum solid model as reference. This 3D reference model is modelled using the same linear elements as in the RVEs, resulting in 691,200 elements, to ensure mesh convergence, $c f$. Figure $7 \mathrm{~b}$. Also note that, the shell model used for comparison is the same as the one used to extract deformation quantities for the RVE analyses in the examples.

To make a quantitative comparison of how well the stress results from the different models can recreate the reference solution, we compute the normalised average squared error between the longitudinal transverse shear stress values from the reference $\tilde{\sigma}_{13}$ and the model $\sigma_{13}$ as

$$
e=\frac{1}{n_{\mathrm{s}}} \sum_{k=1}^{n_{\mathrm{s}}}\left(\frac{\tilde{\sigma}_{13}\left(x_{3}^{k}\right)-\sigma_{13}\left(x_{3}^{k}\right)}{\left|\tilde{\sigma}_{13}\right| \max }\right)^{2}
$$

where $n_{\mathrm{s}}$ is the number sampling points through the thickness and $\left|\tilde{\sigma}_{13}\right|^{\text {max }}$ is the maximum absolute value of the reference.

In the following graphs, the resulting stresses are plotted as connecting lines between the values at each Gauss point level and the values have been normalised with respect to the (maximum) value of the reference 3D solution.

\subsection{Isotropic cantilever beam}

A first comparison between the stress components obtained using the different models is made using an isotropic material with a Young's modulus of $70.7 \mathrm{GPa}$ and a Poisson's ratio of zero. We motivate the choice of setting 


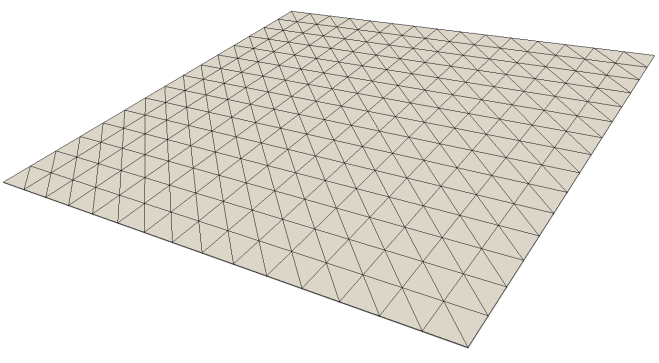

(a)

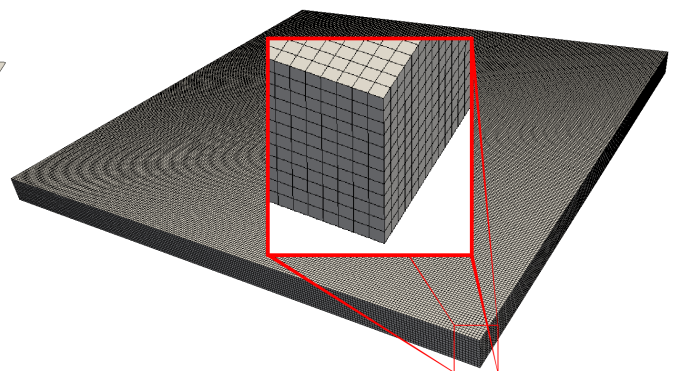

(b)

Figure 7: FE mesh for the shell model (a) and the reference 3D model (b).

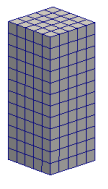

(a)

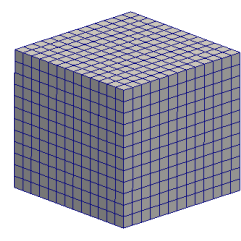

(b)

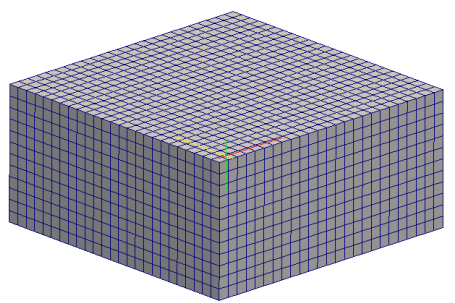

(c)

Figure 8: $1.25 \mathrm{~mm}(\mathrm{a}), 3.25 \mathrm{~mm}(\mathrm{~b})$ and $6.25 \mathrm{~mm}$ (c) in-plane size of the mesoscopic RVE. 
Poisson's ratio to zero by the fact that it allows us to make comparisons with Euler-Bernoulli beam theory to which the reference 3D model, although piecewise linear in $\sigma_{13}$, as expected compares very well, $c f$. Figure 9.

\subsubsection{Assessment of the multiscale method}

Figure 9 shows results for the Mixed boundary conditions applied on the RVE. The variations of the in-plane stress component $\sigma_{11}$ through-thethickness are similar between the shell, solid and the RVE models. Furthermore, the Mixed boundary condition on the RVEs fulfils the traction free condition on the top and bottom surface. However, the through-thethickness variation of the $\sigma_{13}$-component clearly differs between the models. Consequently, although the shape of the variation of the $\sigma_{13}$-component in the RVE shows qualitative improvement with respect to the beam theory as the size increases, an increasing RVE size does not lead to convergence towards the reference model. Instead, the error measure $e$, as described in Eq. (19), increase upon an increase in the RVE size, cf. Table 1. On the other hand, smaller RVE sizes do converge, but towards the shell results.

The resulting bending moment and shear force can be obtained by integrating the stress components in Figure 9 over the thickness; these are displayed in Figure 10 as a function of RVE size. As seen from the figure, an increase in RVE size leads to a decrease in the resultant shear force. This implies that for an increased RVE size, an increased deviation in the applied shear deformation (on the RVE) is obtained compared to both the reference $3 \mathrm{D}$ and the shell model.

Based on the unsatisfying results obtained for Mixed boundary conditions above,we find it interesting to investigate the effects from applying the more constraining types of boundary conditions Dirichlet and Taylor. These results are presented in Figure 11, where we take the 12x13x13 element sized RVE as reference for all RVE sizes. As seen from the figure, Dirichlet or Taylor boundary conditions results in stresses comparable to the shell analysis. Also, by studying the resultant bending moment and shear force, displayed in Figure 12, we can further confirm that by applying the Dirichlet or Taylor boundary conditions the deformation mode of the shell is better captured since the resultants very well match those obtained from the shell analysis.

\subsubsection{Assessment of the stress recovery method}

Shear stress values computed using the stress recovery method, introduced in Section 4, are presented as bullets in Figure 9 and 11. The errors, 


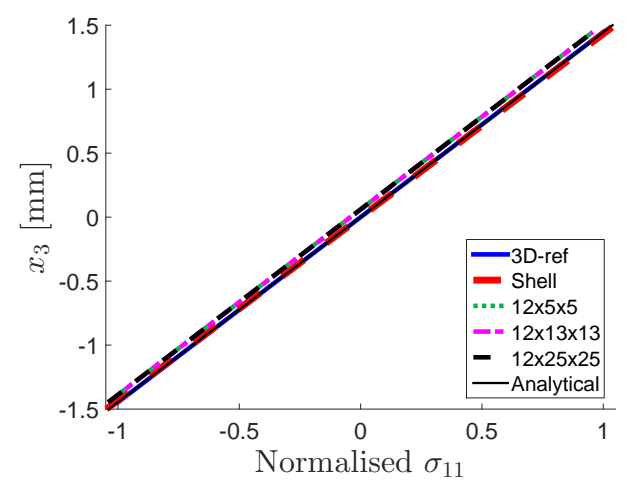

(a)

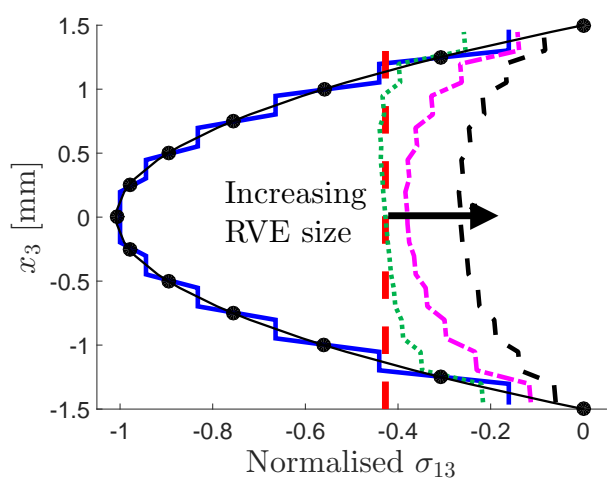

(b)

Figure 9: Longitudinal in-plane normal (a) and out-of-plane shear (b) stress components in the shell and reference 3D models and three different RVE sizes for isotropic material. The black solid line represent the analytical beam solution and the $\bullet$ marks the interface stress values computed using stress recovery.

according to Eq. (19), of the stress produced by this and the other models compared to the analytical solution are summarised in Table 1. For this example, the recovery method is clearly the most capable in reproducing the through-the-thickness variation of the stress found in the analytical solution.

\subsection{Laminated cantilever beam}

Using the same geometry, mesh and load, as in the example above, we have also studied a $\left[ \pm 45^{\circ}\right]_{3 \mathrm{~S}}$ laminate, having one element per ply in the thickness direction for the RVE and for the reference 3D model. The plies are modelled as transversely isotropic, with material properties given in Table 2 .

\subsubsection{Assessment of the multiscale method}

In Figure 13 and 14, the resulting in-plane $\left(\sigma_{11}, \sigma_{12}\right)$ and out-of-plane $\left(\sigma_{13}\right)$ stress components are plotted for the reference 3D model, the shell model and three different RVE sizes using the Mixed boundary condition.

As can be seen in Figure 13, the results from the shell and the RVEs show the same in-plane stress components as that of the reference 3D model. However, as in the isotropic case, the through-the-thickness variation of the out-of-plane shear stress $\sigma_{13}$, displayed in Figure 14, become smoother as the RVE size increases. Also, the results obtained with smaller RVE sizes 


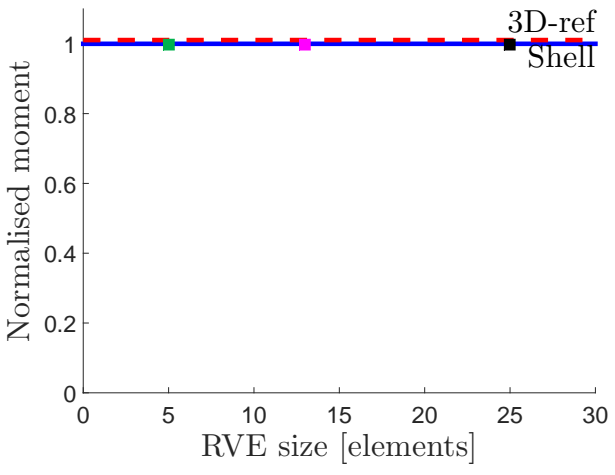

(a)

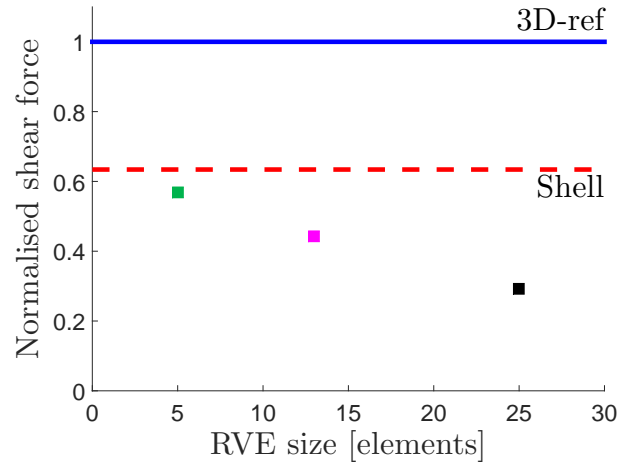

(b)

Figure 10: Normalised resultant moment (a) and shear force (b) in the shell and reference 3D models and three different RVE sizes for isotropic material.

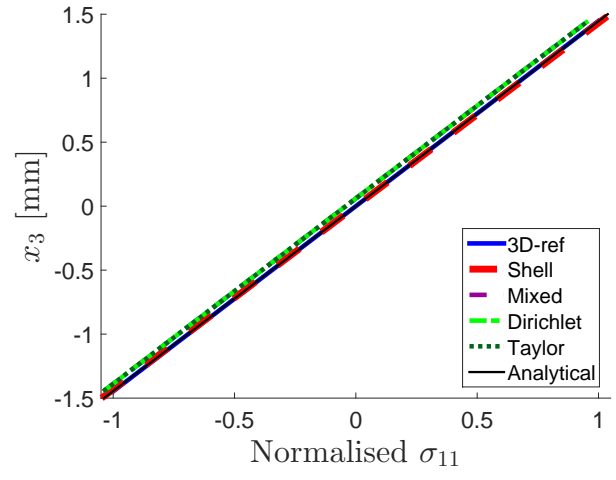

(a)

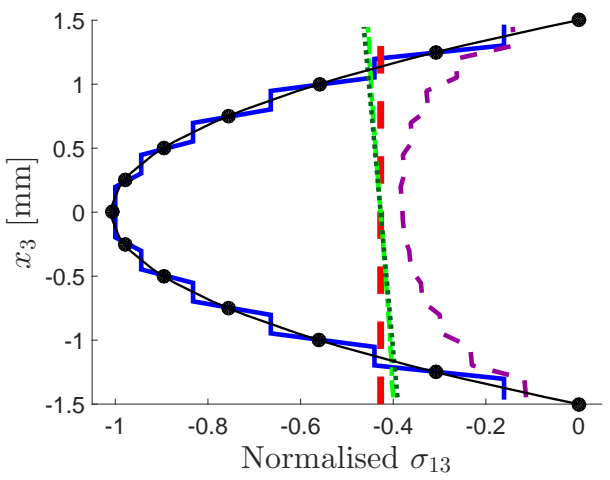

(b)

Figure 11: Effect of boundary condition type on the longitudinal in-plane normal (a) and out-of-plane shear (b) stress components in the RVE (isotropic material). The black solid line represent the analytical beam solution and the $\bullet$ marks the interface stress values computed using stress recovery. 


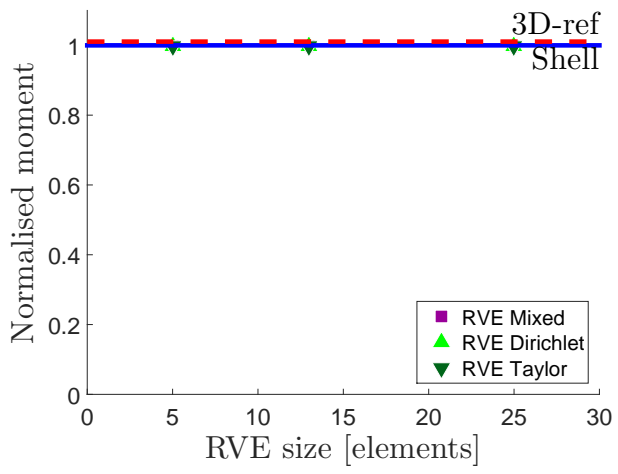

(a)

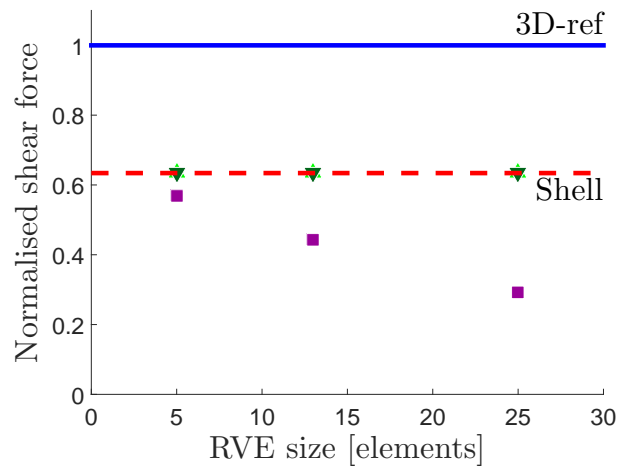

(b)

Figure 12: Normalised resultant moment (a) and shear force (b) for different boundary conditions (isotropic material).

Table 1: Errors in $\sigma_{13}$, according to Eq. (19), compared to the analytical solution of a cantilever beam made of isotropic material.

\begin{tabular}{l|c} 
Model & $e$ \\
\hline 3D-ref & 0.003 \\
Shell & 0.157 \\
RVE 12x5x5 Mixed & 0.143 \\
RVE 12x13x13 Mixed & 0.187 \\
RVE 12x13x13 Dirichlet & 0.151 \\
RVE 12x13x13 Taylor & 0.151 \\
RVE 12x25x25 Mixed & 0.281 \\
Stress recovery & 0.000
\end{tabular}


Table 2: Material properties for laminate model.

\begin{tabular}{cccccc}
\hline $\boldsymbol{E}_{\mathbf{L}}$ & $\begin{array}{c}\boldsymbol{E}_{\mathbf{T}} \\
{[\mathbf{G P a}]}\end{array}$ & $\boldsymbol{\nu}_{\mathbf{L T}}$ & $\boldsymbol{\nu}_{\mathbf{T T}} \mathbf{G P a}^{\prime}$ & $\begin{array}{c}\boldsymbol{G}_{\mathbf{L T}} \\
{[\mathbf{G P a}]}\end{array}$ & $\begin{array}{c}\boldsymbol{G}_{\mathbf{T T}}{ }^{\prime} \\
{[\mathbf{G P a}]}\end{array}$ \\
100 & 10 & 0.25 & 0.25 & 5.0 & 4.0 \\
\hline
\end{tabular}

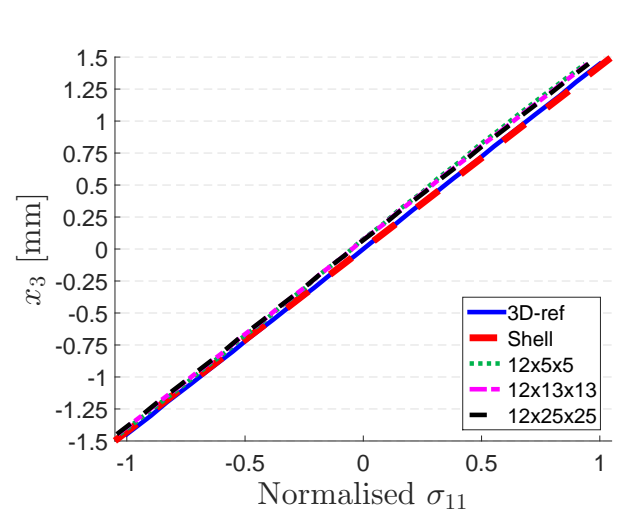

(a)

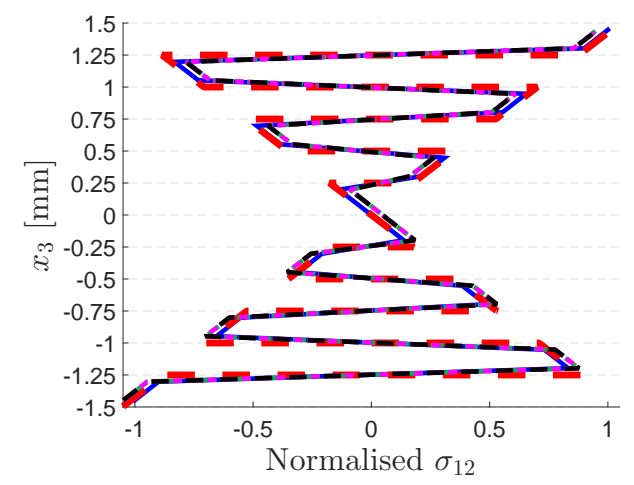

(b)

Figure 13: Relevant in-plane stress components in RVEs of different sizes compared to the shell and reference 3D models when simulating with orthotropic material.

converge towards the shell results. The resulting moment and shear force, displayed in Figure 15, confirm the conclusions drawn from the isotropic case, i.e. that the deformation mode from the macroscopic shell is not maintained in the RVE.

\subsubsection{Assessment of the stress recovery method}

Similar to the isotropic case, the interface shear stress values computed using the stress recovery method show very good correlation with the reference solution, $c f$. Figure 14. This is confirmed by the error measure $e$ in Table 3 where, in particular, the error obtained with the stress recovery method relative to the $3 \mathrm{D}$ reference solution is less than 0.000 .

\section{Conclusions}

In this contribution we have investigated the potential of using two concepts for obtaining a better prediction of the through-the-thickness distribution of the transverse (shear) stresses in equivalent single-layer shell analyses. 


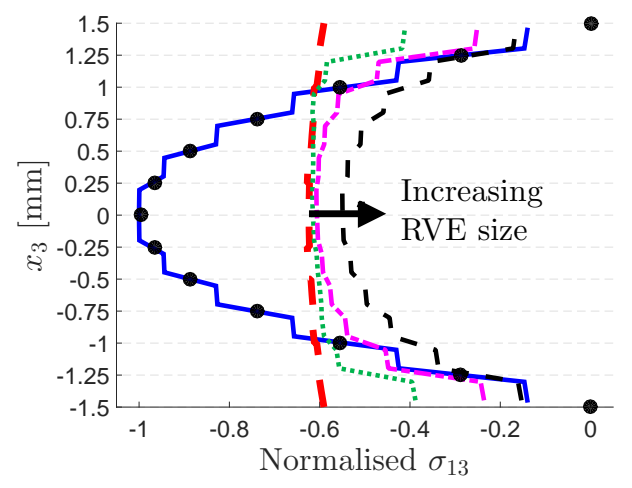

Figure 14: Longitudinal out-of-plane shear stress component in RVEs of different sizes compared to the shell and reference 3D models when simulating with orthotropic material. The $\bullet$ marks the interface stress values computed using stress recovery.

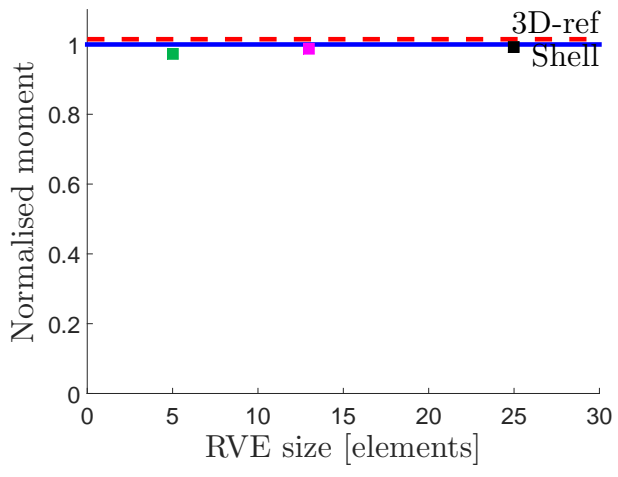

(a)

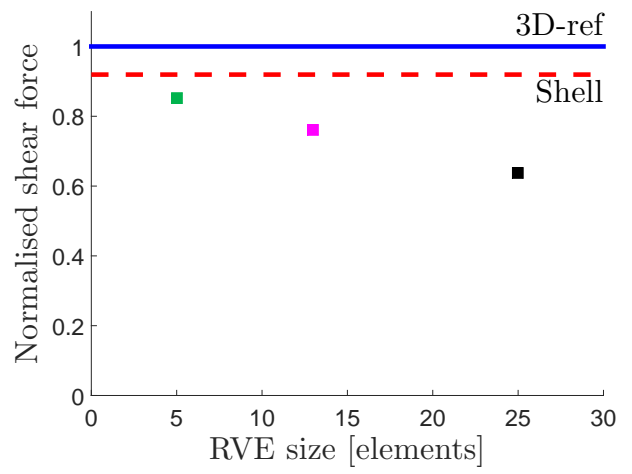

(b)

Figure 15: Normalised resultant moment (a) and shear force (b) in the shell and reference 3D models and three different RVE sizes for orthotropic material. 
Table 3: Errors in $\sigma_{13}$, according to Eq. (19), compared to the 3D reference solution in an angle-ply laminated cantilever beam.

\begin{tabular}{l|c} 
Model & $e$ \\
\hline Shell & 0.112 \\
RVE 12x5x5 Mixed & 0.067 \\
RVE 12x13x13 Mixed & 0.060 \\
RVE 12x25x25 Mixed & 0.088 \\
Stress recovery & 0.000
\end{tabular}

As a first alternative, the multiscale (or rather two-scale) procedure introduced by Larsson and Landervik [9] to model and simulate heterogeneous material structures in thin-walled applications was investigated, where we focused on the prolongation condition ( $c f$. Section 3). Besides following the procedure in [9], where a combination of Dirichlet and Neumann (Mixed) boundary conditions were applied to the RVE, we have also considered pure Dirchlet boundary conditions.

For the Mixed boundary condition, the results show, regardless of material type, that as the size of the RVE increases the resulting through-thethickness stress variation becomes smoother. However, at the same time, the magnitude is decreased compared to the reference model. In our numerical examples this means that, although the shape better resembles the reference $3 \mathrm{D}$ model, the deformation mode of the RVE is diverging from the one on the macroscale. Therefore we conclude that the problem with the proposed type of expansion and boundary conditions, in that the average deformation is not guaranteed across the scales, cannot be neglected even though energy equivalence is maintained. We believe this to be an interesting finding since similar boundary conditions have been adopted also by other authors, although normally with in-plane Periodic Boundary Conditions (PBCs), cf. $[21,22,23]$. We emphasise however that not even in the case of PCBs, deformation equivalence across the scales can be guaranteed. The consequences of this are uncertain since the mentioned papers do not discuss e.g. transverse shear or the effect of a varying RVE-size.

To ensure deformation equivalence across the scales we also investigated the effect of applying pure Dirichlet boundary conditions in the prolongation phase. However, the results achieved from this correspond to what is obtained 
in the shell analysis and no improvements, with respect to accuracy of stress prediction, can be observed.

The conclusions drawn above were not an apparent problem in [9], where porous material was analysed, however to directly apply their approach to simulate laminated FRP plates is not straightforward. To obtain results closer to the reference 3D model with the given method, deformation equivalence across the scales is probably necessary. This would require the expansion of the relative placement, described in Subsection 3.1, to be of higher order.

On the positive side, we can conclude that the proposed stress recovery method very well captures the through-the-thickness stress variation in our presented examples, provided that the stress field is made continuous over element boundaries. The stress recovery method is rather cheap, and if the only goal is to improve the prediction of the transverse stresses in analyses of laminated plates, we conclude it to be more suitable compared to a multiscale approach.

What we propose for future work on ESLM analysis of progressive failure in laminates is thereby to adopt a post-processing procedure where the first step is a smoothing of the in-plane stress components over neighbouring elements, followed by an integration of the transverse stress components using the $3 \mathrm{D}$ equilibrium equations. We conclude that it is then not a necessity to use a very advanced layer-wise shell element formulation, or a very detailed through-thickness spatial FE resolution, to obtain a sufficient accuracy of stresses needed for the prediction of e.g. delamination initiation. Instead, a rather low order shell element can be used if proper post-processing of the stresses is made.

We consider these findings interesting in the sense that computational efforts can be saved by using an ESLM approach with a less expensive shell formulation. Indeed, the post-processing step would require additional computational effort, but the interval at which it is done could be controlled. It would for most applications be sufficient to perform the recovery at predefined time instants, and not within every time increment. This is in contrast to the adoption of a more advanced LWM, in which case the additional computational effort is present throughout the simulation.

In the long perspective, we suggest to combine the stress recovery method with e.g. the modelling concept based on the eXtended Finite Element Method (XFEM) proposed by Brouzoulis and Fagerström [24], which is designed to allow for the modelling of arbitrarily many delaminations within a single shell 
element. Thereby, the modelling would be based on a ESLM approach, using a less advanced shell element formulation for saving computational cost, which is then dynamically enriched in areas where delamination initiation (or propagation) is predicted by the suggested post-processing method. This way any number of delaminations can be included in the simulation.

\section{Acknowledgements}

The current work has been funded by the Swedish Strategic Vehicle Research and Innovation Programme on Vehicle and Traffic Safety via the project Modelling crash behaviour in future lightweight composite vehicles Step 1 which is gratefully acknowledged.

\section{References}

[1] ERTRAC. European Roadmap Safe Road Transport. Technical report, European Road Transport Research Advisory Council, 2011.

[2] J.N. Reddy. Mechanics of Laminated Composite Plates: Theory and Analysis. Boca Raton, CRC Press, 1997.

[3] Erasmo Carrera. A priori vs. a posteriori evaluation of transverse stresses in multilayered orthotropic plates. Composite Structures, 48(4):245-260, 2000 .

[4] Erasmo Carrera. Developments, ideas, and evaluations based upon Reissner's Mixed Variational Theorem in the modeling of multilayered plates and shells. Applied Mechanics Reviews, 54(4):301, jul 2001.

[5] Ireneusz Kreja. A literature review on computational models for laminated composite and sandwich panels. Open Engineering (formerly Central European Journal of Engineering), 1(1):59-80, 2011.

[6] Maria Cinefra, Claudia Chinosi, Lucia Della Croce, and Erasmo Carrera. Refined shell finite elements based on RMVT and MITC for the analysis of laminated structures. Composite Structures, 113:492-497, apr 2014.

[7] Erasmo Carrera. Historical review of Zig-Zag theories for multilayered plates and shells. Applied Mechanics Reviews, 56(3):287-308, may 2003. 
[8] J.Tinsley Oden and Kumar S. Vemaganti. Estimation of Local Modeling Error and Goal-Oriented Adaptive Modeling of Heterogeneous Materials. Journal of Computational Physics, 164(1):22-47, 2000.

[9] Ragnar Larsson and Mats Landervik. A stress-resultant shell theory based on multiscale homogenization. Computer Methods in Applied Mechanics and Engineering, 263:1-11, may 2013.

[10] V. Kouznetsova, M.G.D. Geers, and W.A.M. Brekelmans. Multiscale constitutive modelling of heterogeneous materials with a gradientenhanced computational homogenization scheme. International Journal for Numerical Methods in Engineering, 54(8):1235-1260, jul 2002.

[11] E.W.C. Coenen, V.G. Kouznetsova, and M.G.D. Geers. Computational homogenization for heterogeneous thin sheets. International Journal for Numerical Methods in Engineering, 83(March):1180-1205, 2010.

[12] Cécile E. Helfen and Stefan Diebels. Computational homogenisation of composite plates: Consideration of the thickness change with a modified projection strategy. Computers \& Mathematics with Applications, 67(5):1116-1129, mar 2014.

[13] R. Rolfes and K. Rohwer. Improved transverse shear stresses in composite finite elements based on first order shear deformation theory. International Journal for Numerical Methods in Engineering, 40:51-60, 1997.

[14] Moinuddin Malik and Ahmed K. Noor. Accurate determination of transverse normal stresses in hybrid laminated panels subjected to electrothermo-mechanical loadings. International Journal for Numerical Methods in Engineering, 47:477-495, 2000.

[15] A.K. Noor and M. Malik. An assessment of five modeling approaches for thermo-mechanical stress analysis of laminated composite panels. Computational Mechanics, 25:43-58, 2000.

[16] T. Kant and B.S. Manjunatha. On accurate estimation of transverse stresses in multilayer laminates. Computers \& Structures, 50(3):351365, 1994. 
[17] Byung Chul Park, Jin Woo Park, and Yong Hyup Kim. Stress recovery in laminated composite and sandwich panels undergoing finite rotation. Composite Structures, 59(2):227-235, 2003.

[18] O.C. Zienkiewicz and J.Z. Zhu. The superconvergent patch recovery and a posteriori error estimates. Part 1: The recovery technique. International Journal for Numerical Methods in Engineering, 33(7):1331-1364, 1992.

[19] Daniel Jose Payen and Klaus-Jürgen Bathe. Improved stresses for the 4-node tetrahedral element. Computers \&3 Structures, 89(13-14):12651273, mar 2011.

[20] Lawrence Bush, Quanling Deng, and Victor Ginting. A locally conservative stress recovery technique for continuous Galerkin FEM in linear elasticity. Computer Methods in Applied Mechanics and Engineering, 286:354-372, jan 2015.

[21] Jörg Hohe. A direct homogenisation approach for determination of the stiffness matrix for microheterogeneous plates with application to sandwich panels. Composites Part B: Engineering, 34(7):615-626, 2003.

[22] J. Gager and H.E. Pettermann. Numerical homogenization of textile composites based on shell element discretization. Composites Science and Technology, 72(7):806-812, 2012.

[23] L. Gigliotti and S.T. Pinho. Exploiting symmetries in solid-to-shell homogenization, with application to periodic pin-reinforced sandwich structures. Composite Structures, 132:995-1005, jul 2015.

[24] Jim Brouzoulis and Martin Fagerström. An enriched shell element formulation for efficient modeling of multiple delamination propagation in laminates. Composite Structures, 126:196-206, feb 2015. 